\title{
Problems of Increasing the Intelligence of Algorithms for Optimal Distribution of the Current Load on the Combined Heat and Power Plant and Ways to Solve Them
}

\author{
Arakelyan Edik ${ }^{1, *}$, Kosoy Anatoliy ${ }^{1}$, Andryushin Alexander ${ }^{1}$, Mezin Sergey ${ }^{1}$, Yagupova Yulia ${ }^{1}$, Leonov Maxim ${ }^{1}$, Pashchenko Fedor ${ }^{2}$ \\ ${ }^{1}$ ACS TP dept., National Research University “MPEI”, Moscow 111250, Russia
}

${ }^{2} V$. A. Trapeznikov Institute of Control Sciences of Russian Academy of Sciences, Moscow 117997, Russia

\begin{tabular}{l} 
A R T I C L E I N F O \\
\hline Article history: \\
Received: 05 July, 2021 \\
Accepted: 16 September, 2021 \\
Online: 29 October, 2021 \\
\hline Keywords: \\
Optimal distribution \\
Thermal and electrical load \\
Complex composition \\
Multi-criteria optimization \\
Dynamic optimization \\
Intelligent functions \\
\hline
\end{tabular}

\section{Introduction}

This article is a continuation of the work originally presented at the international conference "Managing the Development of Large-scale Systems" (MLSD-20) [1]. In this report, the problems and increasing the intelligence of algorithms for optimal load distribution at a thermal power plant were considered in the following conditions:

- the optimal distribution of the time-constant load of the station with a given composition of generating equipment was considered, i.e. the problem of static optimization;

- a single-criteria task was solved according to the economic criterion - the maximum profit of the station from the sale of electricity in various sectors of the electricity market and power and heat to the thermal consumer, or minimizing the total fuel consumption at the station.

The technical capabilities of automation complexes are several generations ahead of the software implementation of applied algorithms for optimal control and control of the technological process of electricity production. It is more rational, in the conditions of high prices for the work of talented programmers and technologists, not to try to solve this problem with the old catching-

${ }^{*}$ Corresponding Author: Arakelyan Edik, Edik arakelyan@inbox.ru up methods, but to approach this problem cardinally, entrusting the solution of the complex problem of the efficiency of electricity generation at thermal power plants to artificial intelligence. The intermediate stage of the transition to the management of the entire plant by $\mathrm{AI}$ is the transition to the management of individual levels, whether it is the level of the station or the level of the power unit. [2]-[4].

Determination of the optimal operating mode for power plants and power systems is an urgent problem in the power industry. Over time, the solution of this optimization problem is being improved and at present, in connection with the transition of energy to market relations, the optimization of power plant operation modes seeks not only to reduce the cost of electricity generated, but also to increase reliability and reduce emissions of harmful fuel combustion products into the environment [4]-[7].

The relevance of considering the reliability factor is due to the fact that the task of choosing the optimal operating modes of thermal power plants (TPP) equipment includes two interrelated tasks: choosing the composition of generating equipment and the distribution of heat and electric load with a known composition of generating equipment. Currently, in accordance with the Regulations of the electricity and power market, the selection of the composition of generating equipment and its coordination with 
the system operator (SO) of the power system is carried out 2-3 days before the operational day, and the station performs load distribution per day when preparing an application for entering the electricity and capacity market "for the day ahead". Due to the fact that the agreed composition of the equipment during optimization calculations and execution of the dispatching schedule of loads cannot be changed, considering the reliability factor in solving the problem becomes obvious and mandatory. For TPPs located near urban buildings, the environmental factor is also important. At the same time, the task is not to ensure emissions within the permissible regulatory limits, but to minimize them as much as possible.

In connection with the above, in the development of the report at the conference, this article discusses the problems and ways to improve the intelligence of the algorithm for optimal distribution of thermal and electrical loads of a combined heat and power plant (CHPP) with a complex composition of equipment with a multicriteria problem statement - in terms of efficiency, reliability and environmental friendliness.

At the same time, in addition to the sections of the load schedule with a constant electrical load in time, the sections with a variable load (the stages of unloading and loading) are also considered. This is due to the fact that the duration of the unloading/loading processes of the station is several hours, while the efficiency indicators of the power units vary within a fairly wide range, which leads to significant additional fuel losses if the optimal sequence of unloading/loading of the power units involved in the process of passing the load failure is not observed.

To assess the amount of computational procedures required, we will consider the stages of preparing and entering the power plant market for electricity and capacity in the market conditions of the power plant. In accordance with the Regulations of the electricity and capacity market, the following stages are considered for managing the operating modes of the CHPP equipment for the operational day:

- selection and approval of the composition of generating equipment for the operational day in order to form the optimal station request for each group of delivery points (GDP);

- drawing up a possible plan for electricity generation with projected volumes of heat output to form the optimal application of the station for participation in the competitive market "for the day ahead" for each GDP conducted by the administrator of the trading network (ATN);

- operational optimization when performing the dispatching load schedule for each GDP - optimal distribution of thermal and electrical loads between the switched-on units with a known composition of the operating equipment;

- optimization of the dispatching load schedule to generate an optimal operational price-accepting request for each GDP for the deviation of the electrical load from the dispatching schedule in the balancing market controlled by the SO.

\section{Statement of a Multi-Criteria Static Problem of Optimal Load Distribution}

Setting a multi-criteria task: for a given total electrical and thermal loads of a CHPP, it is necessary to determine such loads at which the total current costs for the production of electrical and thermal energy will be minimal with the greatest reliability of the equipment and the least amount of harmful emissions into the environment [8].

The mathematical formulation of a static problem with three criteria is formed as follows: find

$$
\left.\begin{array}{l}
F_{1}(x)=\sum_{\mathrm{j}=1}^{n} f_{1 \mathrm{j}}\left(p_{j}, q_{j}\right) \rightarrow \min _{\mathbf{x}} \\
F_{2}(x)=\sum_{\mathrm{j}=1}^{n} f_{2 \mathrm{j}}\left(p_{j}, q_{j}\right) \rightarrow \min _{\mathbf{x}}, \\
F_{3}(x)=\sum_{\mathrm{j}=1}^{n} f_{3 \mathrm{j}}\left(p_{j}, q_{j}\right) \rightarrow \min _{\mathbf{x}}
\end{array}\right\}
$$

under the following conditions:

$$
\begin{gathered}
\sum_{j=1}^{n} p_{j}=P_{\Sigma}, \sum_{j=1}^{n} q_{j}=Q_{\Sigma} \\
p_{j_{\text {min }}}<p_{j}<p_{j_{\text {max }}} \\
q_{j_{\text {min }}}<q_{j}<q_{j_{\text {max }}} \\
p_{j} \neq q_{j} \leq S_{j_{\text {max }}} j=1,2, \ldots, n
\end{gathered}
$$

where $F_{1}(x), F_{2}(x), F_{3}(x)$ - optimality criteria for efficiency, reliability and ecology; $f_{1 j}\left(p_{j}, q_{j}\right), f_{2 j}\left(p_{j}, q_{j}\right), f_{3 j}\left(p_{j}, q_{j}\right)$ energy characteristics of the $j$-th power unit, reflecting fuel consumption, average damage for unreliability of operation (due to emergency downtime and failures) and the amount of emissions of harmful substances into the environment; $p_{j}, q_{j}$ - electrical and thermal power of the $j$-th unit; $P_{\Sigma}, Q_{\Sigma}$ - total required electrical and thermal power set for the entire system; $p_{j \min }, p_{j \max }, q_{j \min }, q_{j \max }, S_{j \max }$ - permissible load limits; $\mathbf{x}=\left\{x_{1}, . ., x_{n}\right\} ; \mathbf{p}=\left\{p_{1}, \ldots, p_{n}\right\} ; \mathbf{q}=\left\{q_{1}, \ldots, q_{n}\right\}$.

The essence of optimization is to achieve a compromise solution for three criteria, with an optimization horizon of one to several days when forming an application for the selection of the composition of the included generating equipment for an operational day in 2-3 days, applications for the market for a day ahead, as well as operational applications for the balancing market. When implementing the dispatching schedule of loads, the number of considered criteria can be changed both in the direction of decrease and in the direction of increase

\section{Statement of the Dynamic Problem of Optimal Load Distribution}

The need to consider the formulation of a dynamic optimization problem is due to the following. As a rule, in the absence of control of the operating modes of power units in automatic mode, if it is necessary to involve several power units in changing the load of the station in order to eliminate the likelihood of non-fulfillment of the specified schedule, all power units begin the procedure for changing the load almost simultaneously, without taking into account their individual characteristics. This 
often leads to significant deviations of the total load of the station from the required one, violation of the required time for the station to reach the specified load, especially when it is necessary to stop or start one or more power units. At the same time, in the absence of a clear schedule of load changes over time at each specific power unit, as a rule, there is an advanced or delayed load change, which leads to additional fuel losses, deviations in power generation from the required dispatch schedule, and other violations (for example, deviation of the permissible load change rates during unloading or loading).

To eliminate these disadvantages, it is necessary to organize such a sequence of unloading/loading of power units from a given initial state to the required final, also known state. It is assumed that for these states, with a known equipment composition, the problem of optimal distribution is solved on the basis of a multicriteria approach, and for their docking with each other, the following conditions must be met:

- the change in the load of the power plant $\Delta N_{P P}\left(\tau_{u / l}\right)$ over time is the sum of the change in the loads of each of the power units $\Delta N_{i}\left(\tau_{u / l}\right)$ for the considered time $\tau_{u / l}$ of unloading/loading the station, taking into account the permissible deviation from the dispatch schedule $\alpha$, i.e.

$$
\Delta N_{P P}\left(\tau_{u / l}\right)=(1 \pm \alpha) \sum_{i=1}^{n} \Delta N_{i}\left(\tau_{u / l}\right),
$$

in this case, the condition below is met at each moment of time

$$
N_{P P \tau}=\sum_{i=1}^{n} N_{i \tau}=\sum_{i=1}^{n}\left(N_{i 0}+\Delta N_{i \tau}\right),
$$

where $N_{P P \tau}$ is the power of the station at the moment $\tau ; N_{i 0}, \Delta N_{i \tau}$ - the power of the $i$-th block before the change in the power of the station and its increment in time.

- the rate of load change at each power unit is within the permissible unloading/loading speed

$$
w_{i u / l}^{\min } \leq w_{u p / l} \leq w_{u p / l}^{\max } ;
$$

- the capacity of each power unit can vary from the initial $N_{i 0}$ to the required final capacity $N_{i F}$ in accordance with the dispatching schedule (it is assumed that the power unit capacity limits are taken into account when distributing the station load before and after the load change).

The features of solving this dynamic problem are as follows:

- the composition of the equipment before and after changing the load of the station is identical, and therefore it makes no sense to consider the criteria of reliability and environmental friendliness, so a single-criteria task is considered;

- as a criterion of two possible functions used in solving similar problems - the total fuel loss or the total fuel consumption during the load change at the station, it is advisable to use the function of fuel consumption during the load change;
- in contrast to the previously adopted methods in this paper, in addition to the fuel costs calculated from the consumption characteristics of each power unit, it is also necessary to take into account the additional fuel costs not taken into account by these characteristics in the unloading/loading processes associated with the unsteadiness of heat exchange processes in heat exchangers, unstable operation of regulators, etc. in transients in the equipment of the unit [9].

Taking into account the additional costs, the optimization criterion is presented in the form:

$$
B_{P P}\left(N_{P P u / l}, \tau_{u / l}\right)=\sum_{i=1}^{n}\left[\int_{\tau_{0}}^{\tau_{u / H I}} B_{i \tau} \delta \tau+\Delta B_{P P i}\left(\frac{\Delta N_{i u / l}}{N_{i}}, W_{i u / l}\right)\right]
$$

where $B_{P P}\left(N_{P P u / l}, \tau_{u / l}\right)$ - the total fuel consumption at the station during the load change; $N_{P P u / l}$ - the magnitude of the load change at the station; $\tau_{u / l}$ - the duration of the load change; $B_{i}$ the function of fuel consumption on the $i$-th block when the load changes; $\Delta B_{P P i}\left(\frac{\Delta N_{i u / l}}{N_{i}}, W_{i u / l}\right)$ - the function of additional costs in the unloading/loading processes, depending on the depth and speed of the load change on the $i$-th block.

The initial data of the task are: the initial and final power of the station, the duration of the load change $\tau_{u / l}$, hour (min.); the rate of load change at the station $W_{P P}=\Delta N_{P P} / \tau_{u / l}$; the energy characteristics of the units in the form of a dependence [8]; the functions of the dependence of additional fuel costs in the unloading/loading processes, the initial and final load distribution of the station before and after the load change between the generating equipment, the permissible unloading/loading speeds of the power units.

The desired (variable) values are: the moment of the beginning of unloading/loading of each power unit in relation to the beginning of the load change at the station; the dynamics of the load change of each power unit during the transition from the initial state of the station to the final state.

To solve this problem, it is proposed to use a dynamic programming algorithm (Bellman's method) [8], which is used to optimize the management of multi-stage processes or to optimize multi-link systems, when it is necessary to find the control action (or the state of each link of the system) at each stage, so that the general control criterion reaches the optimal value. At the same time, at each next stage, the result of optimization at the previous stage is taken into account. The peculiarity of the method consists in a sequential, step-by-step solution of the optimization problem, so that at each stage only a relative number of possible options are selected that provide the optimal values of the selected criterion.

\section{Ways to Increase Intelligence in Solving the Optimal Distribution Problem Based on Multi-criteria Optimization}

The analysis of the above statements of static and dynamic optimization shows the complexity of their solution by traditional 
methods and it becomes obvious that their practical implementation will require the use of modern information and digital technologies. Below are some, far from exhaustive, ways to increase the intelligence of the automated control system in solving the tasks set.

- Transition to the principle of advanced (predictive) control when performing the dispatching schedule of loads. The essence of the proposal: first, based on the analysis of previous optimization calculations, to estimate the required delay time between the start of the optimization calculation and before the formation of the impact on the power units. There are two possible ways to implement it: 1) calculate it based on the average data in the chain - the task, the preparation of the source data, the solution, the solution check, the signal generation, the controller, the actuator; 2) create a database at the specified time and, if necessary, taking into account the dimension of the current optimization problem, find a suitable lead time;

- Before performing optimization calculations, determine the feasibility and necessity of selecting the number of criteria and, if there is more than one criterion, the priority of the criteria. The assessment can be carried out on the basis of expert assessments, based on the current load area on the daily schedule according to pre-accepted conditions (the significance of the criteria at different hours of the day, or by the amount of load). For example, when passing through the failures of load schedules, the environmental criterion can either be excluded from consideration, or can be taken into account as a restriction, while the significance of the remaining two criteria (efficiency and reliability) depends on the redundancy mode: if all units are in generation mode, then the efficiency criterion is more significant; if there are stopped units, then the reliability criterion. When passing the maximum load, the efficiency criterion plays a secondary role, because all units operate in an economical mode.

The development of the library module of the intelligent controller is inseparable from the problem of verification and validation of the results obtained. We can get an ideal imitator of a traditional PID controller, we can get an auto-tuning blog simulator based on linear regression models, or we can just get an intelligent controller with a defect in a hidden area of knowledge. The flaws in the training of the received intelligent controller may be hidden from us for many years of operation, and then, for reasons that we do not understand, the optimizer will go into this dark area of uncalculated or incorrectly calculated.

Developers are already presenting many options for really working intelligent controllers based on deep neural networks and machine learning principles, and technical capabilities allow you to scale and launch impressive neural network controllers in terms of complexity. But so far, there are problems of proving that these regulators find and follow the global extremum, which is an uncritical problem at this stage, and the problem of proving the stability and reliability of the operation of this intelligent regulator in the time and operational areas of determination.

So far, they are trying to hide the problem of this fundamental limitation behind the no less cumbersome problem of creating a digital twin of a power plant. It is assumed that we will have a mathematical model similar to a full-scale simulator of the station. And on this model, we will first, but in real time, check the solutions of the intelligent controller before making adjustments to the controller of a real object. This is an interesting, but not entirely honest approach. Removing the issue of validation and verification of a developed and trained intelligent controller by justifying that everything is checked through a digital double generates, with due responsibility, questions about the validation and verification of a digital double. And if we take into account that the power plant as a control object changes over time - and here we get the problem of the impossibility of building a working model of a digital double without using artificial neural network technology. This again leads us to the problem of trust and the implication of the module's operation and proves the dead-end nature of this way of solving the problem.

At this stage, the solution will be a hierarchical distribution of the entire control object into functional groups, and then a distribution according to the importance and danger of violations of the technological process in each group. From this partition, we will get the area of permissible rights for the intelligent controller module. All these rights should be limited from above by the control of the human operator. [10,11].

To implement the interaction described above, the software that runs the optimal load balancing algorithm must support a number of functions and features, which are presented below:

- Support multiple standard data transfer protocols: OPC UA, OPC DA, HART, Modbus, etc.

- Buffering, storing and analyzing information in a separate archive according to the selected parameters for a specified period of time. This information includes: key parameters of the unit operation, digitized consumption characteristics obtained online and via the regulatory and technical documentation.

- The ability to automatically obtain approved dispatch schedules that serve as similar data for the implementation of the calculation.

- Generation of reports and graphs based on the received data with an assessment of the degree of accuracy of the problem solution.

- Implementation of the authentication process, with login only for registered accounts. This is necessary to restrict access and prevent unauthorized changes to the logic of the program.

- Development taking into account cross-platform, in connection with the current trends in the industrial market.

The requirements described above are currently met by most modern PTCs, including domestic ones. This means that for the technical implementation of this algorithm and its integration into the automated process control system of the station will not require the purchase and adjustment of new equipment. It is enough to virtually isolate the unused power of the PTC, creating a virtual functional node for solving intellectual tasks. In this node, everything is also virtual, that is, without spending money on the purchase and laying of new cable connections, to bring existing signals from sensors and programs from the PTC. The output signals from the intelligent optimization node can be easily connected both directly with additional signals in the automated regulation systems routines of the block capacities, and in the form of recommendation information on the mnemonic circuits to the operator. 
5. Problems of Integration of the Optimal Load Distribution Algorithm into the PTC Application Software and Possible Solutions

The analysis of the functional capabilities of modern automated control systems (ACS) of TPP built on the basis of PTC of both domestic and foreign production shows that the improvement of the quality of technological processes, optimization of their modes and parameters, based on new technical and software tools and achievements of the modern theory of automatic control of technological processes, occurs mainly at the level of equipment and power unit, and to a much lesser extent - at the level of power plants. It is obvious that the further development of the ACS through the intellectualization of power plant process control systems should be aimed at creating a single system for optimal control of technological and production processes in all operating modes of equipment, power units and the plant as a whole. The analysis of the current state of use in the design of ACS of domestic software and hardware complexes, their information and functional capabilities in terms of the possibility of integration into their application software of the proposed optimal distribution algorithm revealed the problems that arise in this case, both from the point of view of technical and information support. When considering the problems that arise, it is assumed that in the future, in addition to the optimal distribution algorithm, a number of other station-level control algorithms will be integrated into the application software of PTC, the implementation of which will significantly increase the overall level of intelligence of the automated control system as a whole.

The main requirements for the technical part, in which the intelligent algorithm will be directly implemented, are: sufficient server power, in which the software complexes will work; high speed of the server and the algorithms; the software must be able to transmit data according to the OPC UA specification, which is the newest and most advanced specification for information transmission (for the first time in the OPC family of technologies, the possibility of cross-platform compatibility was implemented). OPC UA also has the highest level of security in the OPC family of technologies. Its main advantage is the transmission of data over the Internet.

There are also basic requirements for systems that are imposed regardless of the software and hardware complex used, such as:

requirements for the protection of system information, including the protection of the system from the penetration of external software and technical influences that are made over the data transmission network;

- all changes to the system access settings must be logged in the system status log;

- access to the system should be divided into roles, for example: engineer, operator, etc.;

- the system should be able to restore databases after failures, failures and accidents.

- Some requirements for storing calculated data:

- Storing parameter values for a period specified by the user or the system;
- Full description of the database where parameter values are stored;

- Long-term storage of information;

- The user should be able to run algorithms and generate reports for any period (hours, minutes, seconds, etc.);

- The system should be able to observe changes in parameters in real time.

According to the prediction of parameters for calculating the power unit's technical and economic indicators (TEI) and carrying out optimal load distribution, the system should be able to select the past periods from the system's database, in the case when the equipment used coincides with the composition of the forecast period. Forecasting allows you to save a lot of time on entering the initial data. If it is impossible to predict the technological parameters of the system, it should be possible to manually enter the initial data by the operator.

The analysis showed that the above requirements are currently met by the majority of modern PTC, including domestic ones. This means that the technical implementation of the proposed algorithms and their integration into the automated process control system of the station will not require the purchase and adjustment of new equipment. It is enough to virtually isolate the unused power of the PTC, creating a virtual functional node for solving intellectual tasks. The output signals from the intelligent optimization node can be easily connected directly as additional signals to the ACS routines of the block capacities, or as recommendation information to the mnemonic circuits to the operator.

The technical difficulties of integrating the developed artificial intelligence modules into the existing software and hardware complexes at the stations do not require, with the proper level of qualification of the station personnel, a planned shutdown of the entire station for repair. Individual modules for adjusting the settings of existing regulators can be exported and activated on running equipment, since initially these modules only give recommendations. The subsequent switching of the output ports of these modules with the input ports of the controller settings can occur both in manual mode and in automatic mode, when the convergence of the expected and received results of the operation of the bundle of the intelligent controller and the digital double of the station is achieved.

And at the stage of a planned shutdown, it is recommended transfer some of the design functions of the automated process control system from the block level to the station level - those tasks that are associated with general station services (for example, calculation and analysis of the power units TEI, selection of the optimal pressure in the condenser), which will significantly relieve the information load of the block level controllers.

\section{Conclusion}

- The studied formulation of a multi-criteria static problem for sections of the load schedule with a constant and a singlecriteria dynamic problem for sections with a variable load with an optimal distribution of the current load at a thermal power plant with a complex composition of equipment. 
- To increase the intelligence of algorithms for optimal load distribution, a number of methods are proposed, including using machine learning and artificial intelligence methods. Approaches to the use of modern machine learning methods to the specifics of the tasks of power distribution of thermal power plants are proposed.

- Possible approaches to the methodology and practical approaches to the technical implementation of the integration of optimization problem algorithms into the PTC application software, their information and technical support, provided that all existing functions of the automated control system are performed simultaneously, are developed.

\section{Conflict of Interest}

The authors declare no conflict of interest.

\section{Acknowledgment}

The work is carried out with the financial support of the Russian Science Foundation, grant No. 19-38-90095.

\section{References}

[1] E.K. Arakelyan et al., "Ways to increase the intelligence of algorithms for optimal load distribution at CHPP with complex equipment composition", Managing the development of large-scale systems (MLSD-2020), 2, 904911, 2020, doi: 10.1109 / MLSD49919.2020.9247651.

[2] E.K. Arakelyan et al., "Approaches to increasing the intelligence of automated control systems of large power plants by solving optimization problems of block and station levels", Managing the development of largescale systems, 2, 434-436, 2018, doi: 10.1109/MLSD.2018.8551935.

[3] Y. Tverskoy, "Features and problems of the current stage of development of the creation technology of automated control system of TPP", Thermal Engineering, 10, 37-44, 2010.

[4] E.K. Arakelyan et al., "Problems of modern automated process control systems based on software and hardware complexes and a possible way to solve them", Vestnik MPEI, 6, 15-20, 2014.

[5] R.E. Steuer, Multiple Criteria Optimization: Theory, Computations, and Application, John Wiley \& Sons, 1986.

[6] Y. Sawaragi, H. Nakayama and T. Tanino. "Theory of Multiobjective Optimization”, Mathematics in Science and Engineering, 1985.

[7] I. Gorlach and O. Wessel, «Optimal Level of Automation in the Automotive Industry» Engineering Letters, 16, 141-149, 2008.

[8] V. Sabanin et al., "Mathematical and software support of the algorithm for correction of measured parameters for calculating technical and economic indicators at a thermal power plant”, Vestnik MPEI, 1, 21-27, 2003.

[9] E.K. Arakelyan et al., Optimization and optimal control, Moscow Publishing House of MPEI, 2008.

[10] E.K. Arakelyan et al., Improving the efficiency and maneuverability of equipment for thermal power plants, MPEI Publishing House, 1993.

[11] E.K. Arakelyan et al., "Evaluation of the optimal level of intelligence of the automated control system of high-power power units based on modern PTC" Managing the development of large-scale systems 5, 574-576, 2019.

[12] I.N. Daimand et al. "Synthesis of intelligent automated control systems for complex technological processes”, Automation in industry, 7, 2013. 